\title{
Research on Short-Circuit Discharge Characteristics of Capacitive Circuits Based on Safety Spark Test Apparatus
}

\author{
Shulin Liu and Yongxiu Zhao \\ School of Electrical and Control Engineering, Xian University of Science \& Technology, Xian 710054, China \\ Correspondence should be addressed to Shulin Liu; 1sigma@163.com
}

Received 26 February 2014; Revised 26 April 2014; Accepted 26 April 2014; Published 26 May 2014

Academic Editor: Her-Terng Yau

Copyright ( 92014 S. Liu and Y. Zhao. This is an open access article distributed under the Creative Commons Attribution License, which permits unrestricted use, distribution, and reproduction in any medium, provided the original work is properly cited.

\begin{abstract}
Based on the safety spark test apparatus, the short-circuit spark discharge (SCSD) characteristics of the simple capacitive circuit and switching converter are studied. It is pointed out that their SCSD process can be divided into four stages, that is, dielectric-breakdown stage, spark-generated stage, spark-maintenance stage, and spark-extinguish stage; moreover, there is different equivalent spark resistance in each stage. For the simple capacitive circuit, its spark resistance is larger and maintaining voltage is almost unchanged in the spark-maintenance stage. For the switching converter, its output short-circuit characteristics depend strongly on the load resistance and its maintaining voltage reduces rapidly with the decrease of the load resistance. The circuit model is proposed, which can simulate the output SCSD process of the switching converter. By using the least-squares method, the relationship expressions between the discharge duration and capacitance in each time-stage are derived and the corresponding equivalent resistance is obtained. The mathematical models are established, and the expressions of the discharge current and voltage are deduced. Experiment and simulation results are positive in the analysis showing the feasibility of the proposed models.
\end{abstract}

\section{Introduction}

Electronic equipment applied in flammable and explosive conditions must meet anti-explosive requirements. Intrinsic safety is the optimal means for anti-explosive requirements. Therefore, the electronic circuit and electrical equipment applied in the explosive and dangerous places are always designed to be intrinsic safety instead of others.

As for research on the intrinsic safety, the electronic circuit is usually divided into the simple capacitive circuit, the simple inductive circuit, and the complex circuit [1]. However, the inductor and the capacitor are the energy storage elements of the electronic circuit or electrical equipment. Once the inductor is disconnected or the capacitor is shortcircuit in the electronic circuits, the produced spark [2-6] from the storage elements may ignite the prescriptive gas or their mixtures $[3,5]$ and cause serious damage [6]. Therefore, it is very important to research the discharge characteristics of the inductor and the capacitor. These researches will help take effective measures to prevent igniting combustible and explosive gas.

At present, there are a lot of researches on the discharge characteristics of the inductive circuit, and the obtained research results [7-9] have important reference value. However, the research reports on the discharge characteristics of the capacitive circuit are less. In $[10,11]$, only the discharge forms of the capacitive circuit were introduced. In [12], the tentative researches on the mathematical expressions of the short-circuit voltage and current for the simple capacitive circuit were reported, but there were great differences between this theoretical analysis and experimental results obtained by using the safety spark test apparatus. In [13], the short-circuit discharge characteristics of a simple capacitive circuit were analyzed, but the influence of the switching converter's load resistance on the discharge characteristics cannot be considered. In $[14,15]$, the discharge characteristics of compound circuits were studied, but the discharge duration was not quantitatively analyzed. 


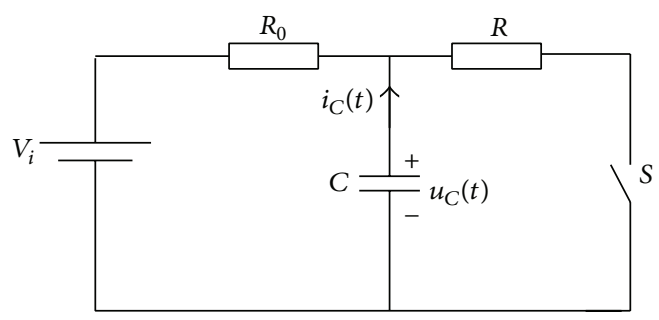

FIGURE 1: Short-circuit discharge principle circuit of a simple capacitive circuit.

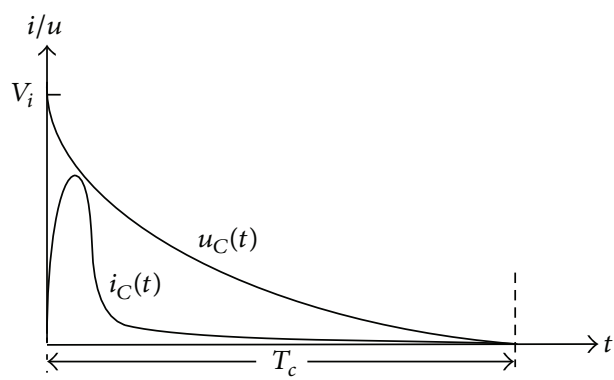

FIGURE 2: Short-circuit discharge waveforms of the simple capacitive circuit with noncontact switch.

As for research on the intrinsic safety problem, the output of the switching converter can be regarded as a capacitive circuit $[16,17]$. However, the output short-circuit discharge process of the switching converter is nonlinear and timevarying, and the related research reports are more rare. In [18], the method is studied to improve the intrinsic safety performance through adding the cut-off type protection. In [19], the output short-circuit spark discharge energy and output intrinsic safety criterion of buck converters are researched. But the influence of capacitance on the discharge duration was not considered in $[18,19]$.

To solve the above problem, the SCSD characteristics of the simple capacitive circuit and switching converter are studied in depth, and a circuit and mathematical models which can simulate the SCSD characteristics are presented in this paper. The influence of the capacitance on the discharge duration will be analyzed.

\section{Short-Circuit Discharge Characteristic of a Simple Capacitive Circuit}

2.1. Short-Circuit Discharge Characteristics of the Capacitive Circuit with Noncontact Switch. The principle circuit researching the SCSD characteristics of the simple capacitive circuit is shown in Figure 1, where $V_{i}$ is the supply voltage, $R_{0}$ is the charging current-limiting resistor, and $R$ is the equivalent resistance of the capacitor and the switch $S$ and lines, usually $R_{0} \gg R$.

Suppose the switch $S$ in Figure 1 is a noncontact switch (such as Transistor, MOSFET). When the circuit is in steady state, the voltage across the capacitor is $V_{i}$. If the switch $S$ is suddenly closed (suppose $t=0$ ), the capacitor-voltage is given by

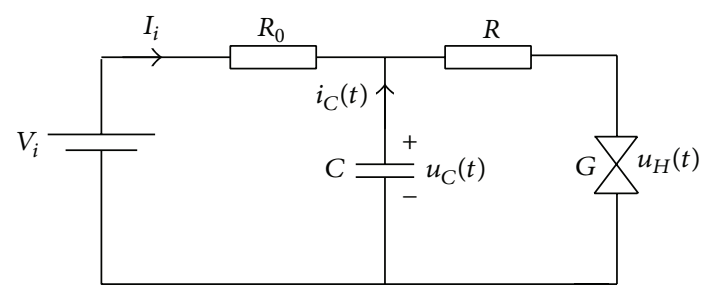

FIGURE 3: Spark discharge experiment circuit of the capacitor.

$$
u_{c}(t)=V_{i} e^{-t / \tau}
$$

where $\tau=R C$, which is the discharge-time constant.

The discharge curve of the capacitor is shown in Figure 2, where $i_{C}(t)$ is the capacitor-current and $u_{C}(t)$ is the capacitorvoltage.

From Figure 2, $i_{C}(t)$ rises rapidly to the maximum when the capacitive circuit with noncontact switch is short-circuit. Then, $i_{C}(t)$ and $u_{C}(t)$ are exponentially decayed and the shortcircuit spark cannot be produced in the capacitive circuit with noncontact switch.

2.2. Short-Circuit Discharge Characteristics of Capacitive Circuit with Contact Switch. Supposing the switch $S$ in Figure 1 is a contact switch (such as relay and two electrodes of the safety spark test apparatus), the short-circuit discharge characteristics are much more complex. Even for the same capacitor and supply voltage, because of the complexity of the discharge process and uncertainty of the short-circuit, each discharge process is not exactly the same. Therefore, a lot of experiments must be done to obtain the typical short-circuit discharge characteristics. The spark discharge experiment circuit based on the safety spark test apparatus is shown in Figure 3, where $G$ is the safety spark test apparatus and $u_{H}(t)$ is the spark discharge voltage.

In the safety spark experiment of the capacitive circuit, the spark test methods are adjusted according to the parameters of the test circuit. For example, in order to guarantee enough charge time, the ignition interval of the tested circuit should be suitably extended by reducing the number of tungsten wires. During the experiment, the short-circuit discharge waveforms are recorded by using TDS3020.

(a) Short-Circuit Discharge Process of the Capacitive Circuit with Contact Switch. When the electrodes of $G$ in Figure 3 are closed, the energy from the power supply and the capacitor are discharged to the electrode gap. Since $R_{0}$ is very large, 


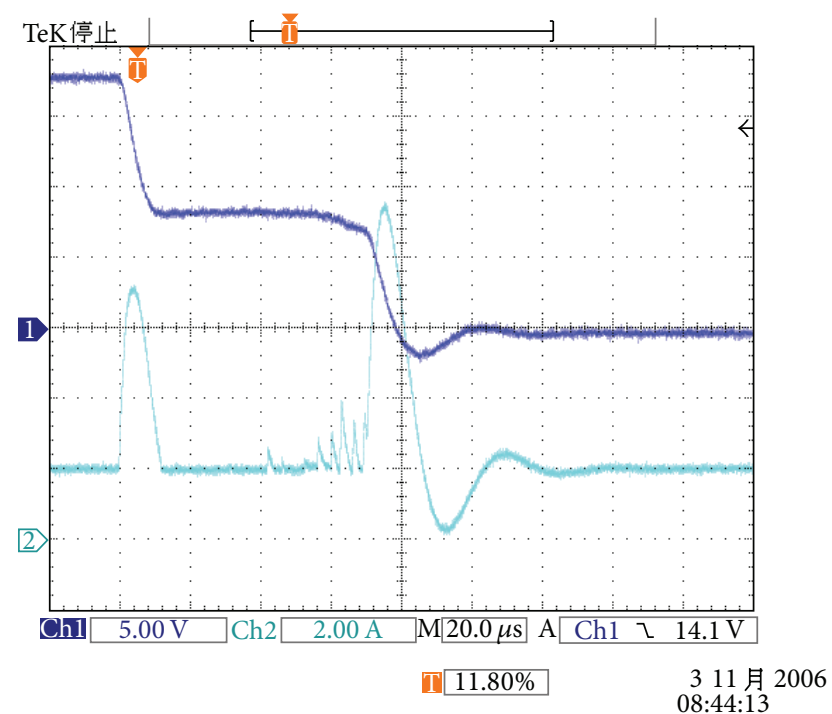

FIGURE 4: Short-circuit discharge waveforms of the capacitive circuit with contact switch $\left(V_{i}=18 \mathrm{~V}, C=8.2 \mu \mathrm{F}\right.$, and $\left.R=560 \Omega\right)$.

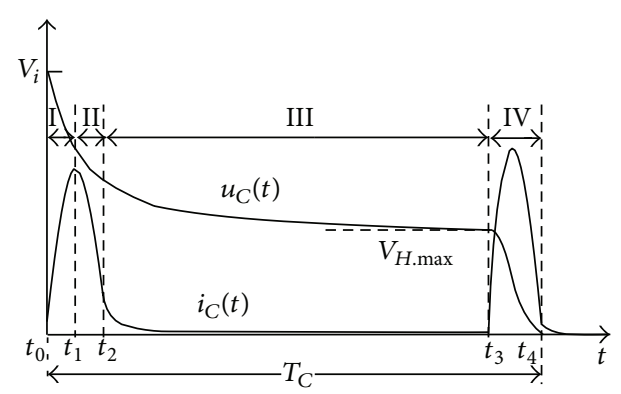

FIGURE 5: Waveforms of the short-circuit discharge in the capacitive circuit.

the impact of the power supply on the capacitor discharge is ignored. The test waveforms are shown in Figure 4.

Through the statistical analysis of a large number of waveforms, the typical discharge current and voltage waveforms are drawn in Figure 5. The SCSD process can be divided into four stages.

Stage I is dielectric-breakdown stage, from $t_{0}$ to $t_{1}$. At the beginning of this stage, the electrodes are separated. When the electrodes start closing $\left(t=t_{0}\right)$, the distance between two electrodes is gradually shrinking. The dielectric is not broken down until the electrode-distance is reduced to the dielectricbreakdown distance. Then, the capacitor-current rises rapidly and the electrode voltage lowers rapidly from the open-circuit voltage. At the end of this stage, the SCSD current reaches the maximum value.

Stage II is spark-generated stage, from $t_{1}$ to $t_{2}$. The SCSD current and voltage of the capacitive circuit rapidly decrease in this stage, till the current decreases to a very small value and the voltage reduces to a relatively stable value. Simultaneously, the discharge spark is generated in this stage.

Stage III is spark-maintenance stage, from $t_{2}$ to $t_{3}$. In this stage, the spark resistance reaches a larger value and is almost unchanged. Only small current flows through the spark discharge circuit. The spark-maintenance voltage of the interelectrodes decreases slowly to the minimum $\left(V_{H \text {,min }}\right.$, typical range is $8 \sim 10 \mathrm{~V})$. The duration of spark discharge depends strongly on the capacitance.

Stage IV is spark-extinguish stage, from $t_{3}$ to $t_{4}$. In this stage, two electrodes are completely closed due to external force at the end of the short-circuit discharge. The electrode voltage drops to zero andthe residual energy stored in the capacitor is released; the second peak of the spark discharge current appears. But the released energy is mainly absorbed by the resistance and electrodes of the capacitive circuit.

(b) Short-Circuit Discharge Characteristics of the Capacitive Circuit with Contact Switch. From Figures 4 and 5, it is found that The SCSD current and voltage have fast change during the short-circuit discharge of the capacitive circuit. In addition, the higher the capacitor-voltage, the greater the peak current of the spark discharge. During the shortcircuit discharge, the released energy is smaller in stage III but larger in other stages. In stage IV, two electrodes are completely closed, and the released energy is mainly absorbed by the resistance and the electrodes. Consequently, the spark discharge energy of the first two stages is the main energy to ignite flammable and explosive gas. The duration in stages I and II is much shorter with respect to the whole discharge process.

According to the above analysis, the release of the shortcircuit discharge energy in the capacitive circuit is strongly concentrated; therefore, it is easy to ignite flammable and explosive gas. For igniting explosive gas, the instantaneous power must be particularly considered besides the spark discharge energy; that is, the short-circuit spark discharge must have enough both large energy and power to ignite flammable and explosive gas. Therefore, when the spark discharge power is smaller, it is hard to ignite the explosive gas, even if the discharge duration is very long (low voltage and large capacitance).

\section{Output Short-Circuit Discharge Characteristics of the Switching Converter}

A switching converter belongs to a typical nonlinear system, which consists of inductor, capacitor, and nonlinear switches; moreover, there are various working states and operating modes. Because the output of a switching converter has a large filter capacitor, it can be regarded as a capacitive circuit from the output of the switching converter, as shown in Figure 6 . Where $R_{L}$ is a load resistance, the other parameters are the same as parameters in Figure 1.

The short-circuit discharge characteristics of the switching converter are more complicated than a simple capacitive circuit, which will be discussed in detail in the following sections.

The explosive experiments are carried out for the output of the switching converter through the spark test apparatus based on IEC standards. Typical output short-circuit discharge current and voltage waveforms of buck converter are shown in Figure 7. 


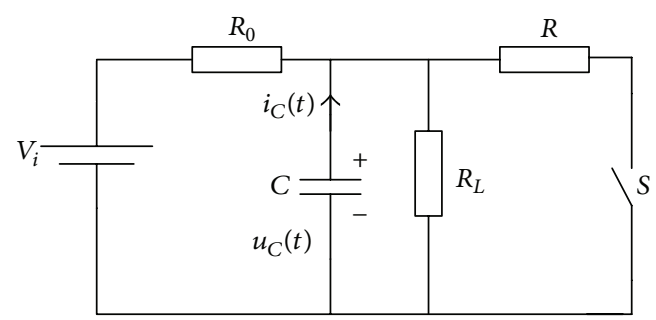

FIGURE 6: Output equivalent circuit of the switching converter.

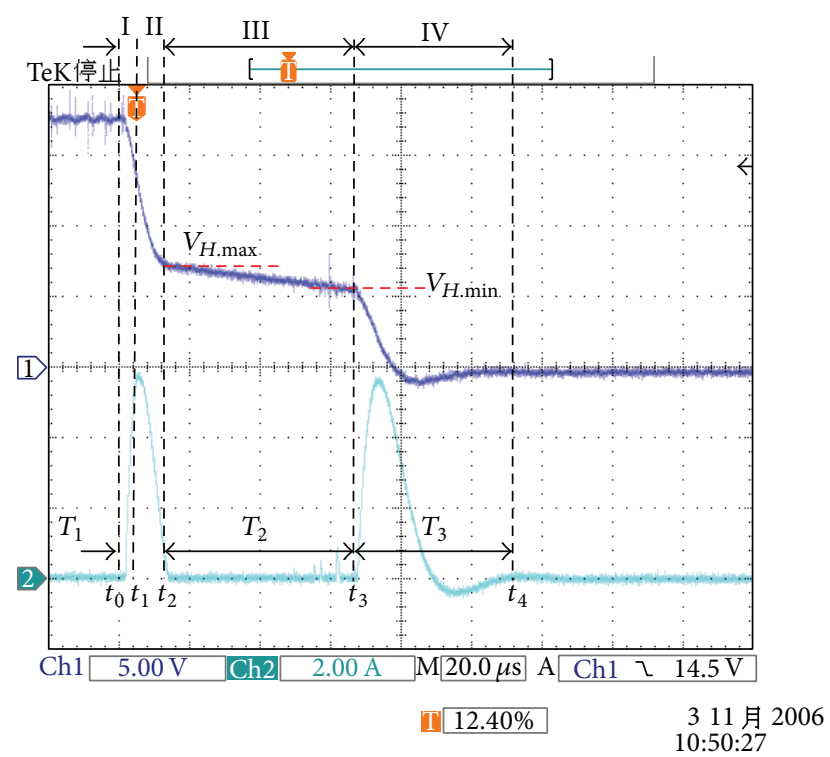

FIGURE 7: Output short-circuit discharge current and voltage waveforms of buck converter.

Comparing Figure 7 with Figure 4, it is found that they have some similarities. The output short-circuit discharge process of the switching converter can be also divided into four stages (shown in Figure 7), that is, dielectricbreakdown stage, spark-generated stage, spark-maintenance stage, and spark-extinguish stage. But there are main differences in spark-maintenance stage and in spark-extinguish stage. Detailed description is as follows.

(1) In the spark-maintenance stage, the reducing speed of the maintaining voltage becomes faster with the decrease of the load resistance. It is mainly caused by a parallel connection of the output capacitor and the load resistance. The spark discharge characteristics may be regarded as an equivalent spark resistance when the spark is produced. From the test waveforms in Figure 7, we can see that the spark discharge current is very small, even if the spark discharge voltage is higher. Thus, the spark resistance is larger and up to about hundreds and even thousands ohm. Because the load resistance is much smaller than the spark resistance, the part energy storied in the capacitor is consumed by the load resistance. This results in the reducing of the maintaining voltage.
(2) In the spark-maintenance, the minimum spark-maintenance voltage also becomes smaller due to the load resistance.

(3) In the spark-extinguish stage, the maximum current decreases because the minimum spark-maintenance voltage becomes smaller.

Therefore, when the output of the switching converter is short-circuit, the output short-circuit current and the minimum maintaining voltage reduce with the decrease of the load resistance. The SCSD characteristics depend strongly on the load resistance.

\section{Modeling Analysis of the Output Short-Circuit Discharge of the Switching Converter}

4.1. Model Circuit of the Short-Circuit Discharge Characteristics. As shown in Figure 7, the output short-circuit discharge characteristics of the switching converter are nonlinear. The spark resistance in each stage is different. In stages I and II it is smaller and larger in stage III.

To get the model circuit simulating the short-circuit discharge process, using $V_{H \text {. max }}$ and $V_{H \text {. min }}$ in Figure 7 as the dividing point, the output short-circuit discharge process of the switching converter can be divided into 3 time-stages, as shown in Figure 7, where the spark resistance in both $T_{1}$ timestage (from $t_{0}$ to $t_{2}$ ) and $T_{3}$ time-stage (from $t_{3}$ to $t_{4}$ ) is very small.

For $T_{2}$ time-stage (from $t_{2}$ to $t_{3}$ ), the spark resistance is in parallel with $R_{L}$. Because the spark resistance is far greater than $R_{L}$, the equivalent spark resistance is approximately equal to the load resistance $\left(R_{L}\right)$.

$T_{1}, T_{2}$, and $T_{3}$ in Figure 7 represent the duration of each time-stage, respectively.

According to the above analysis, a model circuit, which can simulate the output SCSD process of the switching converter, is proposed, as shown in the dotted box in Figure 8. In Figure 8, $T_{1}$ and $R_{1}, T_{2}$ and $R_{L}$, and $T_{3}$ and $R_{3}$ are the discharge duration and the equivalent resistance (the sum of $R$, spark resistance, and load resistance) in 3 time-stages, respectively; $V_{i}$ is the initial capacitor-voltage; $D$ is a diode; $S_{1}$ and $S_{2}$ are the noncontact switches; $V_{H \text {.min }}$ and $V_{H \text {.max }}$ are the minimum and maximum spark-maintenance voltage, respectively; $u_{C}(t)$ and $i_{C}(t)$ are the capacitor-voltage and capacitor-current during the discharge process, respectively.

Each current-flowing loop in Figure 8 can simulate the spark discharge process corresponding to 3 time-stages $\left(T_{1}\right.$, $T_{2}$, and $T_{3}$ ). The principle of the proposed model circuit is described as follows.

(1) $T_{1}$ time-stage: when $u_{C}(t)>V_{H \text {. max }}, D$ is conductive, and $S_{2}$ is shutdown. The discharge current flows through both $R_{1}$ and $R_{L}$. Due to $R_{L} \gg R_{1}$, where $R_{1}$ is the equivalent resistance of the capacitor and the switch $S$ and line, the current flowing through $R_{L}$ can be ignored. Therefore, the discharge resistance is approximately equal to $R_{1}$, which is very small. 


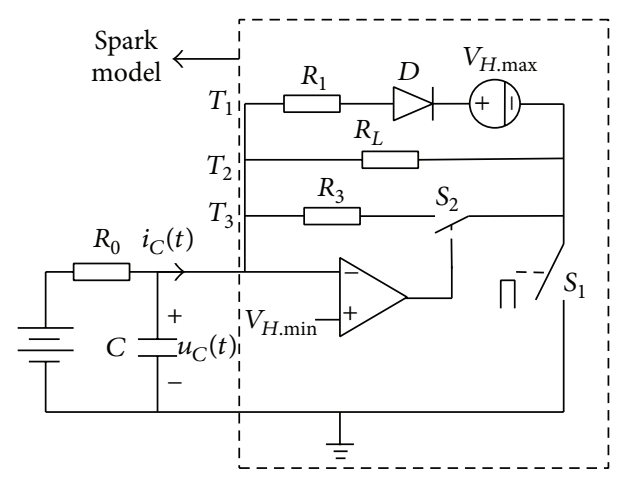

Figure 8: Model circuit of short-circuit discharge process.

(2) $T_{2}$ time-stage: when $V_{H \text {. min }}<u_{C}(t)<V_{H \text {. max }}, D$ is cut-off, and $S_{2}$ is still in shutdown. The discharge current is only through $R_{L}$, and the discharge resistance is $R_{L}$.

(3) $T_{3}$ time-stage: when $u_{C}(t)<V_{H \text {. min }}$, the comparator produces a high level, $S_{2}$ is on, and $D$ continues to be cut-off. The discharge current flows through both $R_{L}$ and $R_{3}$. Owing to $R_{L} \gg R_{3}$, where $R_{3}$ is the equivalent resistance on the line, the discharge resistance is approximately equal to $R_{3}$, which is also very small.

The above analysis shows that the proposed model circuit may simulate the whole output short-circuit discharge process of the switching converter.

4.2. Parameters Determination of the Equivalent Circuit. In the $T_{1}$ time-stage, supposing that the initial value $\left(V_{i}\right)$ and end value $\left(V_{H . \max }\right)$ of the capacitor-voltage $\left(u_{C}(t)\right)$ are known, as soon as the relationship between $T_{1}$ and the circuit parameters is obtained, the value of $R_{1}$ can be determined, and then the discharge characteristic expressions can be derived.

In the $T_{2}$ time-stage, $u_{C}(t)$ decreases linearly from $V_{H \text {. max }}$ to $V_{H \text {.min }}$. To obtain the expressions of the discharge characteristics, the relationship between $T_{2}$ and the circuit parameters must be also found out.

In the $T_{3}$ time-stage, two electrodes are completely closed. Therefore, the discharge characteristics can be regarded as the short-circuit discharge of a capacitive circuit with noncontact switch. $R_{3}$ is the sum of all equivalent resistance, such as the capacitor, the lines, and the internal resistance of spark test apparatus.

In order to obtain the mathematic model of the output short-circuit discharge of the switching converter, the expressions of the duration $\left(T_{1}\right.$ and $\left.T_{2}\right)$ with the external parameters must be found out.

There are many factors to affect the discharge duration, where the capacitance and the initial capacitor-voltage are the most important factors. Only the impact of these two
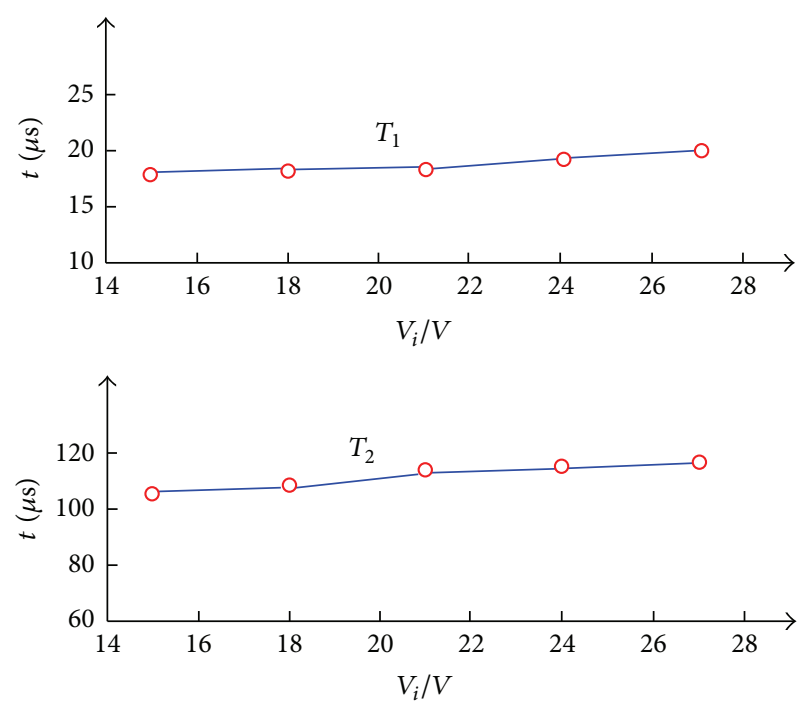

FIGURE 9: Discharge duration versus initial capacitor-voltage.

factors on the discharge duration is discussed in the following section.

(a) Impact of Initial Capacitor-Voltage on the Discharge Duration. The short-circuit spark test is made by using the same capacitor $(16.8 \mu \mathrm{F})$ and different initial voltage. For each initial voltage, 10-time tests are carried out and then get the mean value of 10 test results; the corresponding relationship between the initial capacitor-voltage and $T_{1}$ (or $\mathrm{T}_{2}$ ) is obtained, as shown in Figure 9.

From Figure 9, we can see that the discharge durations $T_{1}$ and $T_{2}$ only slightly increase when the initial capacitorvoltage increases from $15 \mathrm{~V}$ to $27 \mathrm{~V}$. For the electrical and electronic equipment, its power supply voltage is not usually too high. So $T_{1}$ and $T_{2}$ can be regarded as independent of the initial capacitor-voltage in the range of $15 \mathrm{~V}$ to $27 \mathrm{~V}$.

(b) Relation between Discharge Duration and Capacitance. The short-circuit spark test is made with different capacitors. For each capacitor, the discharge test is carried out for 10 times. And their average value is used as the final discharge duration. At last, the corresponding relationship between the duration $\left(T_{1}\right.$ and $\left.T_{2}\right)$ and the capacitance can be obtained. The experimental curves are shown in Figure 10 (where $V_{i}=$ $18 \mathrm{~V}$ ). From Figure 10, we can see that the discharge duration becomes longer with the increase of the capacitance, and the two parameters are approximately linear relationship.

(c) Determination of the Discharge Duration. From the above analysis, the impact of the initial capacitor-voltage on the discharge duration may be ignored. The discharge duration depends mainly on the capacitance and the discharge duration and the capacitance are approximately linear. Therefore, the linear function can be used as the curve fitting. Suppose

$$
T_{1}(C)=a_{0}+a_{1} C
$$




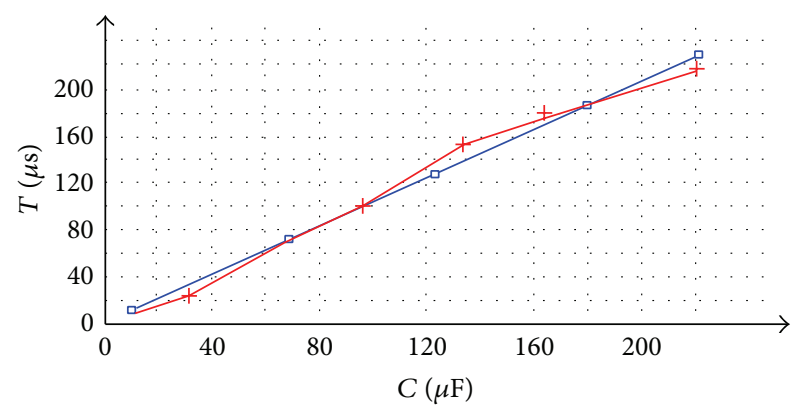

+ Experimental curve

- Fitting curve

(a) $T_{1}$ versus capacitance

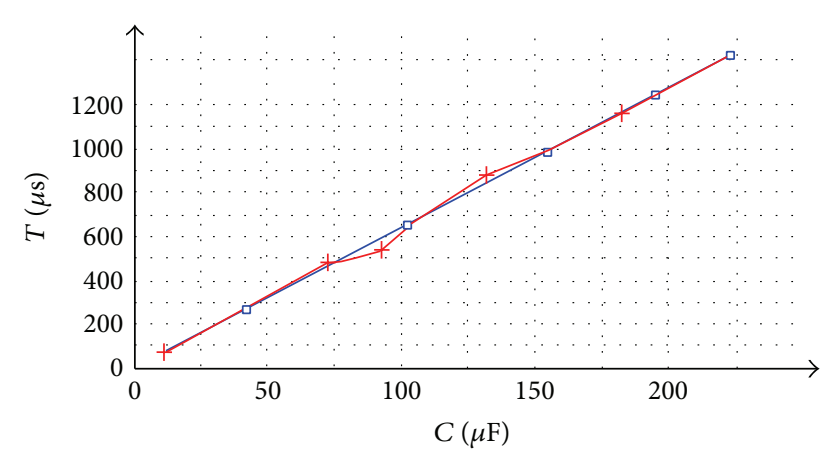

+ Experimental curve

- Fitting curve

(b) $T_{2}$ versus capacitance

FIGURE 10: Discharge duration versus capacitance.

In (2), $a_{0}$ is the intersection of the capacitance function curve and time axis, $a_{1}$ is a slope of the function curve, and $C$ is the capacitance.

Through using the least-squares method of the curve fitting, suppose the sum of error's square is

$$
I=\sum_{i=0}^{n}\left[T_{1}\left(C_{i}\right)-T_{i}\right]^{2}=\sum_{i=0}^{n}\left[a_{0}+a_{1} C_{i}-T_{i}\right]^{2} .
$$

In (3), when $C=C_{i}(=0,1, \ldots, n), T_{i}$ is the experimental data of $T_{1}$ and $n$ is experiment time for different capacitance, so the fitting curve of $T_{1}(C)$ is transformed into the problem solving the minimum value of multivariate function.

Letting $\partial I / \partial a_{0}=0$ and $\partial I / \partial a_{1}=0$, the linear equations about $a_{0}$ and $a_{2}$ are given by

$$
\begin{gathered}
2 \sum_{i=0}^{n}\left(a_{0}+a_{1} C_{i}-T_{i}\right)=0, \\
2 \sum_{i=0}^{n}\left(a_{0}+a_{1} C_{i}-T_{i}\right) C_{i}=0 .
\end{gathered}
$$

Taking 19 groups of experimental datum, thus $n=18$, we can obtain

$$
\begin{gathered}
\sum_{i=0}^{18} T_{i}=19 a_{0}+a_{1} \sum_{i=0}^{18} C_{i}, \\
\sum_{i=0}^{18} T_{i} C_{i}=a_{0} \sum_{i=0}^{18} C_{i}+a_{1} \sum_{i=0}^{18} C_{i}^{2} .
\end{gathered}
$$

Substituting the experimental data into (5), we can obtain

$$
\begin{gathered}
19 a_{0}+1966.7 a_{1}=2029.5 \\
1966.7 a_{0}+288678.59 a_{1}=297720 .
\end{gathered}
$$

Thus, $a_{0}=2.35, a_{1}=1.02$, so

$$
T_{1}(C)=2.35+1.02 C
$$

In (7), the unit of $C$ is $\mu \mathrm{F}$ and the unit of $T_{1}$ is $\mu \mathrm{s}$.

Note that the discharge duration should be zero in $C=0$, but $T_{1} \neq 0$ in (7). In actual circuit, the capacitance is not zero; therefore this does not affect its application. So (7) is feasible.

Similarly, the relationship expression between $T_{2}$ and $C$ is

$$
T_{2}(C)=-6.63+6.40 C \text {. }
$$

Through testing and simulating, the fitting curve is very close to the experimental curves, as shown in Figure 10.

\section{Mathematical Model of the Output Short-Circuit Discharge Characteristics of the Switching Converter}

5.1. Mathematical Analysis of the Output Short-Circuit Discharge Characteristics of the Switching Converter. From the above analysis, the whole output short-circuit discharge characteristics of the switching converter can be described through mathematical expressions. As shown in Figure 7, in the $T_{1}$ time-stage, the duration is $T_{1}$, and capacitorvoltage reduces rapidly from the initial voltage $\left(V_{i}\right)$ to sparkmaintenance voltage $\left(V_{H_{\text {max }}}\right)$. At the same time, capacitorcurrent rises rapidly to the maximum value and then drops quickly to the minimum value (close to zero). Because of $R_{L} \gg R_{1}$, the equivalent resistance is approximately equal to $R_{1}$. According to the above analysis, $u_{C}\left(T_{1}\right)$ is given by

$$
u_{C}\left(T_{1}\right)=V_{i} e^{-T_{1} /\left(R_{1} C\right)}=V_{H \cdot \max } \cdot
$$

Combined with (7) and (9), $T_{1}$ and $R_{1}$ are given as follows:

$$
\begin{gathered}
T_{1}=-R_{1} C \ln \frac{V_{H \cdot \max }}{V_{i}}=2.35+1.02 C \\
R_{1}=-\frac{2.35+1.02 C}{C \ln \left(V_{H \cdot \max } / V_{i}\right)}=-\frac{1}{\alpha C} .
\end{gathered}
$$

In (11), $\alpha=\left(\ln \left(V_{H \cdot \max } / V_{i}\right) /(2.35+1.02 C)\right)$. 
According to (9) (11) and $i_{C}(t)=C\left(d u_{C}(t) / d t\right)$, the capacitor-voltage and capacitor-current can be expressed as

$$
\begin{gathered}
u_{C}(t)=V_{i} e^{\alpha t}, \\
i_{C}(t)=-\alpha C V_{i} e^{\alpha t},
\end{gathered}
$$

where "-" means that the capacitor is in discharge.

In the $T_{2}$ time-stage, the discharge resistance is far greater than the load resistance $R_{L}$, so the equivalent resistance is very close to the load resistance $R_{L}$.

In this stage, the change of the capacitor-voltage is in the range of $V_{H \text {. max }}$ to $V_{H \text {. min }}$, and the short-circuit discharge voltage curve of the capacitive circuit can be approximately regarded as a linear decrease. The spark current is quickly dropped from the initial value at $t_{2}$ moment to a very small value (approximate zero). Experiment results show that the decreasing speed of the spark current is approximately the same as that of the $T_{1}$ time-stage. Thus, the expressions of capacitor-voltage and capacitor-current are given by

$$
\begin{gathered}
u_{C}(t)=\frac{V_{H \cdot \min }-V_{H \cdot \max }}{T_{2}} t+V_{H \cdot \max }, \\
i_{C}(t)=-\alpha C V_{H \cdot \max } e^{\alpha t} .
\end{gathered}
$$

$T_{3}$ time-stage is spark-extinguish stage. According to the analysis of Section 4.2, the expressions of capacitor-voltage and capacitor-current are given by

$$
\begin{aligned}
& u_{C}(t)=V_{H \cdot \min } e^{-t / R_{3} C}, \\
& i_{C}(t)=\frac{V_{H \cdot \min }}{R_{3}} e^{-t / R_{3} C} .
\end{aligned}
$$

In (14), $R_{3}$ is the total resistance of the discharge circuit of the safety spark test apparatus after two electrodes are closed. Usually, $R_{3}$ is $0.5 \Omega$.

5.2. Mathematical Modeling of the Output Short-Circuit Discharge Characteristics of the Switching Converter. According to (9) (14), the mathematical model of the output shortcircuit discharge characteristics of the switching converter can be given as follows (supposing initial moment $t=0$ ).

(1) When $0 \leq t \leq 2.35+1.02 C$, the voltage and current of the capacitor are expressed as

$$
\begin{gathered}
u_{C}(t)=V_{i} e^{\alpha t}, \\
i_{C}(t)=-\alpha C V_{i} e^{\alpha t},
\end{gathered}
$$

where $\alpha=\left(\ln \left(V_{H . \max } / V_{i}\right) /(2.35+1.02 C)\right)$.

(2) When $2.35+1.02 C<t \leq-4.28+7.42 C$, the voltage and current of the capacitor are given by

$$
\begin{gathered}
u_{C}(t)=\frac{V_{H \cdot \min }-V_{H \cdot \max }}{T_{2}}\left(t-T_{1}\right)+V_{H \cdot \max }, \\
i_{C}(t)=-\alpha C V_{H \cdot \max } e^{\alpha\left(t-T_{1}\right)} .
\end{gathered}
$$

(3) When $t>-4.28+7.42 C$, the voltage and current of the capacitor are expressed as

$$
\begin{aligned}
& u_{C}(t)=V_{H \cdot \min } e^{-(t+4.28-7.42 C) / R_{3} C}, \\
& i_{C}(t)=\frac{V_{H \cdot \min }}{R_{3}} e^{-(t+4.28-7.42 C) / R_{3} C} .
\end{aligned}
$$

\section{Verification of the Short-Circuit Discharge Mathematical Model}

To verify the obtained mathematical model, the main parameters are $V_{i}=18 \mathrm{~V}, C=8.2 \mu \mathrm{F}, V_{H \cdot \max }=9 \mathrm{~V}, V_{H \cdot \min }=7 \mathrm{~V}$, $R_{3}=0.5 \Omega$, and $t_{0}=10 \mu \mathrm{s}$ (the initial moment of the short-circuit). According to (15) (17), the voltage and current expressions of the capacitor in each time-stage are given as follows, respectively.

(1) In $T_{1}$ time-stage, $t_{0} \leq t \leq 10.7140+t_{0}$,

$$
\begin{gathered}
u_{C}(t)=18 e^{-0.0647\left(t-t_{0}\right)}, \\
i_{C}(t)=9.5497 e^{-0.0647\left(t-t_{0}\right)} .
\end{gathered}
$$

(2) In $T_{2}$ time-stage, $10.714+t_{0}<t \leq 56.564+t_{0}$,

$$
\begin{gathered}
u_{C}(t)=\frac{-2}{45.85}\left(t-10.174-t_{0}\right)+9, \\
i_{C}(t)=4.7749 e^{-0.0647\left(t-10.174-t_{0}\right)} .
\end{gathered}
$$

(3) In $T_{3}$ time-stage, $t>56.564+t_{0}$,

$$
\begin{gathered}
u_{C}(t)=7 e^{-0.244\left(t-56.564-t_{0}\right)}, \\
i_{C}(t)=14 e^{-0.244\left(t-56.564-t_{0}\right)} .
\end{gathered}
$$

Using the established mathematical model to simulate the output short-circuit discharge characteristics of the switching converter, the output short-circuit discharge curve can be obtained, as shown in Figure 11.

Additionally, the output short-circuit discharge experiments of the switching converter are done by using the safety spark test apparatus based on IEC standard. The experimental waveforms of voltage and current are shown in Figure 7.

From Figures 11 and 7, the spark-maintenance duration is about $45 \mu \mathrm{s}$. However, the discharge peak-current obtained in the experiments is smaller. The main reason is that the elements in the mathematical model are regarded as the ideal components, and some parasitic parameters of the circuit are ignored, such as the parasitic parameters in the circuit components of actual test and parasitic resistances on the line. After these factors are considered, the simulation waveforms can agree well with the experimental waveforms in Figures 11 and 7. It is illustrated that the proposed mathematical model is accurate and feasible.

\section{Conclusions}

(1) Comparing with the noncontact capacitive circuit, the short-circuit discharge process of the capacitive circuit with contact switch is more complicated, where the SCSD can be produced. 


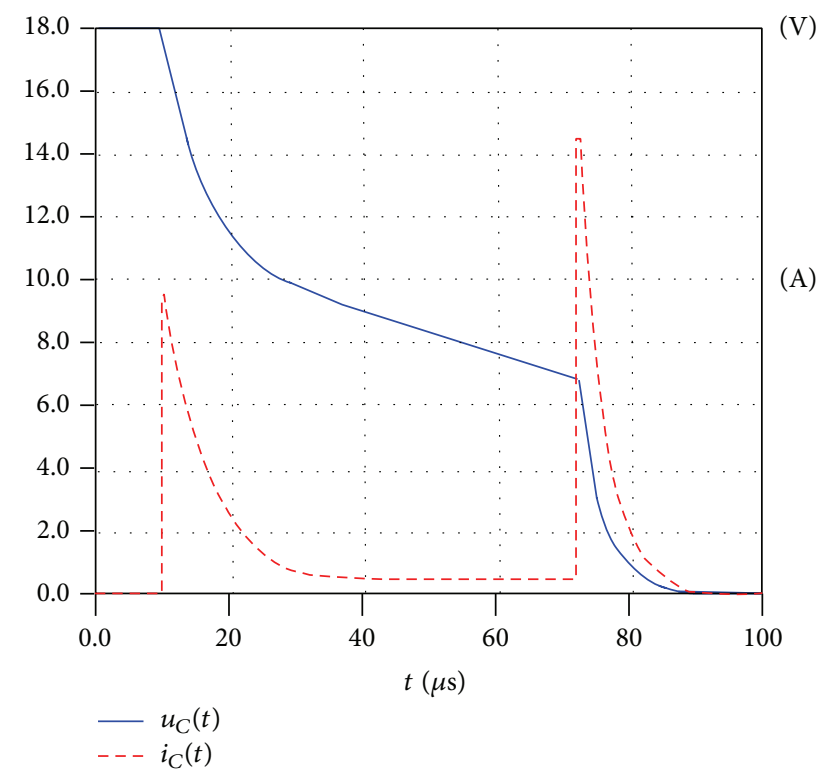

FIGURE 11: The simulation waveforms of mathematical model.

(2) As for a simple capacitive circuit with contact switch and the switching converter, the short-circuit discharge process can be divided into four stages: dielectric-breakdown stage, spark-generated stage, spark-maintenance stage, and spark-extinguish stage.

(3) In the spark-maintenance stage, the spark discharge current is smaller, and there is a larger equivalent spark resistance. As for a simple capacitive circuit, the maintaining voltage is almost constant. However, the maintaining voltage reduces rapidly with the decrease of the load resistance for the switching converter. At the same time, the smaller the load resistance, the lower the minimum maintaining voltage.

(4) The spark discharge energy during the dielectric breakdown stage and sparks production stage is larger and is also the main energy to ignite the flammable and dangerous mixture.

(5) The output short-circuit discharge process can be divided into 3 time-stages in the establishing of mathematical analysis model. The discharge duration becomes longer with the increase of the capacitance in each time-stage, but it is unrelated to the initial capacitor-voltage.

(6) The proposed model circuit can simulate the output short-circuit discharge process of the switching converter. Equivalent discharge resistance is obtained in each time-stage. Moreover, equivalent resistance is smaller in the $T_{1}$ and $T_{3}$ time-stages, while it is larger in the $T_{2}$ time-stage.

(7) The proposed mathematical models can simulate the short-circuit discharge process of the switching converter. The analytic expressions of the current and voltage are obtained. The simulation and experimental results show that the established model can simulate the output short-circuit discharge characteristics of the switching converter.

The obtained conclusions can provide theoretical guidance for designing the intrinsic safety switching converter.

\section{Conflict of Interests}

The authors declare that there is no conflict of interests regarding the publication of this paper.

\section{Acknowledgment}

This work is supported by the National Natural Science Foundation of China $(50977077,51277149)$.

\section{References}

[1] Y. M. Zhang and W. J. Li, Design of Intrinsically Safe Circuit, China Coal Industry Publishing House, Beijing, China, 1992.

[2] Z. C. Wu, X. J. Zhang, and Y. Z. Hu, Gas Discharge, National Defence Industry Press, 2012.

[3] D. Oancea, D. Razus, V. Munteanu, and I. Cojocea, "High voltage and break spark ignition of propylene/air mixtures at various initial pressures," Journal of Loss Prevention in the Process Industries, vol. 16, no. 5, pp. 353-361, 2003.

[4] P. Seers, Spark Ignition: An Experimental and Numerical Investigation, The University of Texas at Austin, 2003.

[5] S. P. M. Bane, Spark Ignition: Experimental and Numerical Investigation with Application to Aviation Safety, California Institute of Technology, Pasadena, Calif, USA, 2010.

[6] W. T. Yin, G. Fu, S. S. Yuan, and J. Y. Dong, "Study on basic characteristics and occurrence regularity of major gas explosion accidents in Chinese coal mines during 2001-2012," China Safety Science Journal, vol. 23, no. 2, pp. 141-147, 2013.

[7] Q. H. Meng, T. L. Hu, and L. H. Mu, "Relationship between discharge time of low energy arc and circuit parameters," Transactions of China Electro-Technical Society, vol. 15, no. 4, pp. 19-21, 2000.

[8] Q.-H. Meng, L.-H. Mu, C.-L. Wang, and J.-H. Liu, "Analysis of low energy arc discharge characteristics based on linear current attenuation model," Journal of China Coal Society, vol. 26, no. 6, pp. 654-656, 2001.

[9] H. N. Zhang, "Research on arc energy relationship of intrinsically safe circuit," Coal Mine Machinery, vol. 32, no. 2, pp. 63-65, 2011.

[10] G. J. Zhang, "Research on ignition of explosive gases by capacitive intrinsic safety short-circuit discharge instantaneous spark," Electric Explosion Protection, vol. 3, pp. 25-28, 2001.

[11] L. J. Mu, "The research about the impact of capacitive discharge in switching intrinsically safe power supply," Vacuum Electronics, vol. 6, pp. 60-63, 2010.

[12] L. H. Zhang, Y. T. Song, and X. Z. Liu, Principle and Application of Intrinsic Safety, China Coal Industry Publishing House, Beijing, China, 1984.

[13] S.-L. Liu, J.-M. Zhong, W.-B. Fan, and M. Zhan, "Short circuit discharge characteristics of the capacitive circuit and its mathematical model," Journal of the China Coal Society, vol. 37, no. 12, pp. 2123-2128, 2012. 
[14] Y. S. Yu, Study on the Theory and Application of the Intrinsically Safe Switch Power Supply, China University of Mining and Technology, 2012.

[15] Y. S. Yu, W. Y. Qi, Y. T. Hu, and X. J. Wu, "Study on discharge characteristics of compound circuits," Coal Mine Safety, vol. 44, no. 9, pp. 54-57, 2013.

[16] S. L. Liu, J. Liu, and Y. L. Yang, "Analysis of output short-circuit discharged energy and optimal design of output intrinsically safe buck converters," in Proceedings of the Asia Pacific Symposium on Safety, pp. 1978-1984, Shaoxing, China, 2005.

[17] S. Liu, J. Liu, and L. Kou, "Intrinsically safe behavior of switching converters and its design," Transactions of China Electrotechnical Society, vol. 21, no. 5, pp. 36-41, 2006.

[18] Y.-S. Yu, W. Zhang, Q.-H. Meng, and X.-J. Wu, "Modeling and analysis on spark discharge of capacitive circuit with cut-off type protection," Journal of the China Coal Society, vol. 38, no. 3, pp. 517-521, 2013.

[19] S. L. Liu, Q. Cui, and Y. Li, "Output short-circuit spark discharging energy and output intrinsic safety criterion of Buck converters," Acta Physica Sinica, vol. 62, no. 16, Article ID 168401, 10 pages, 2013. 


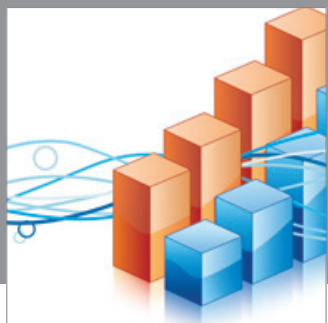

Advances in

Operations Research

mansans

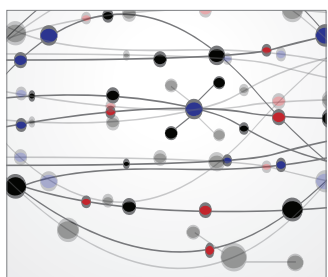

The Scientific World Journal
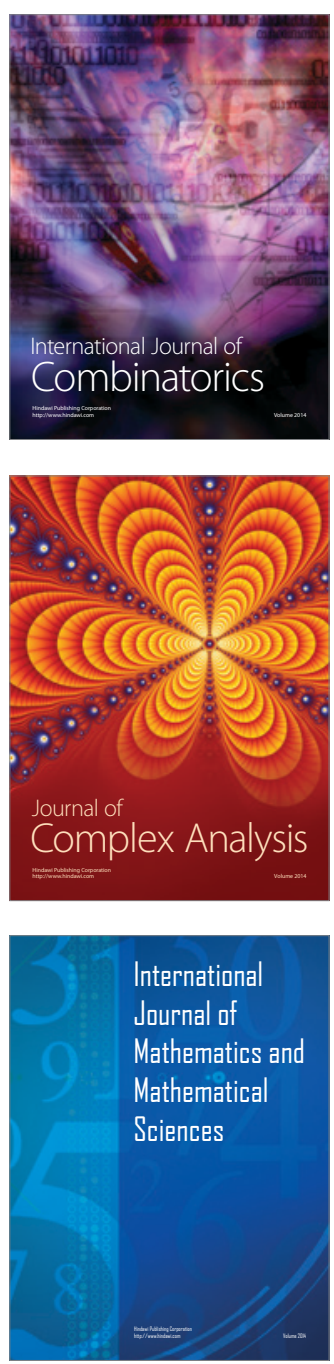
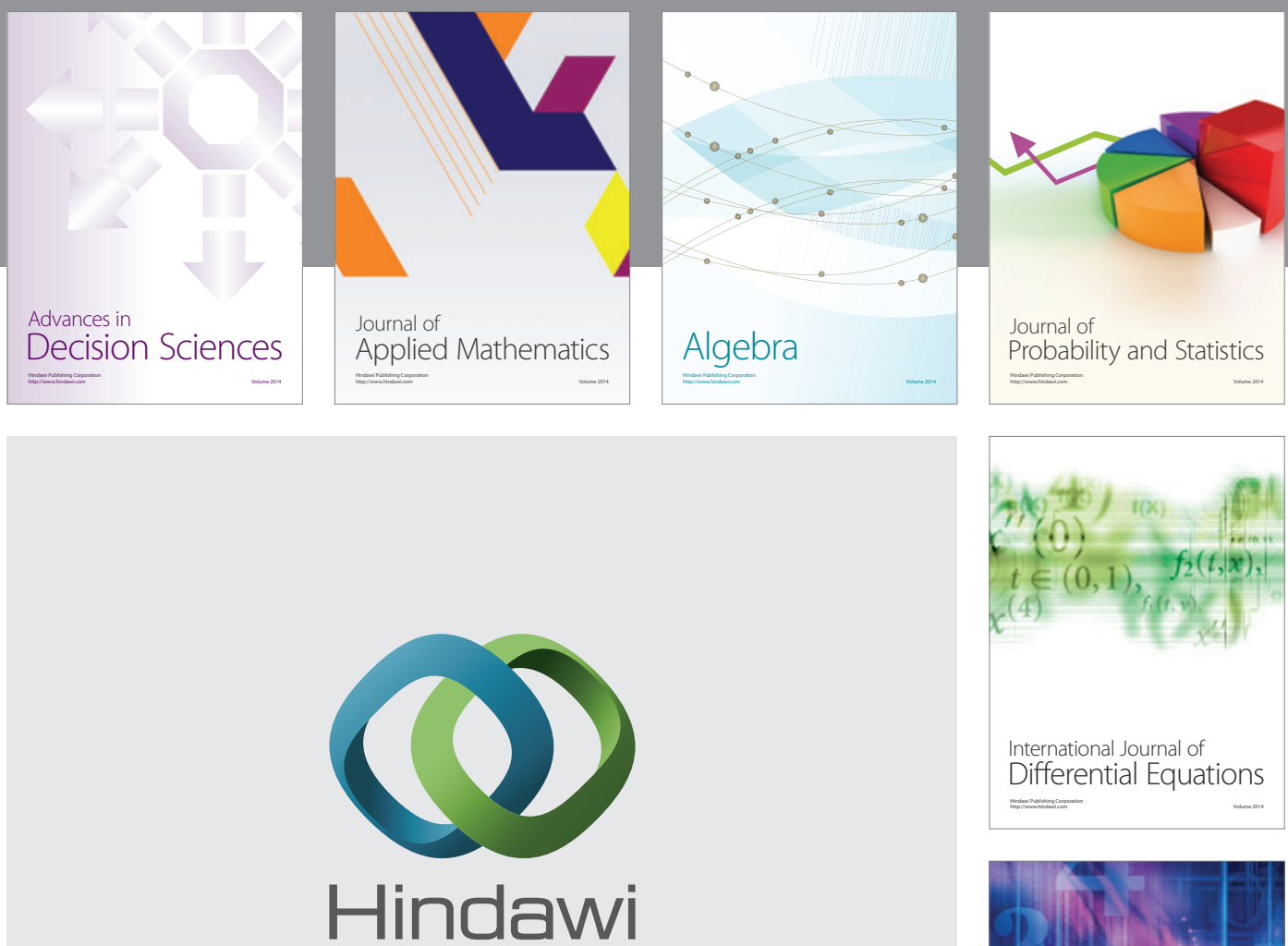

Submit your manuscripts at http://www.hindawi.com
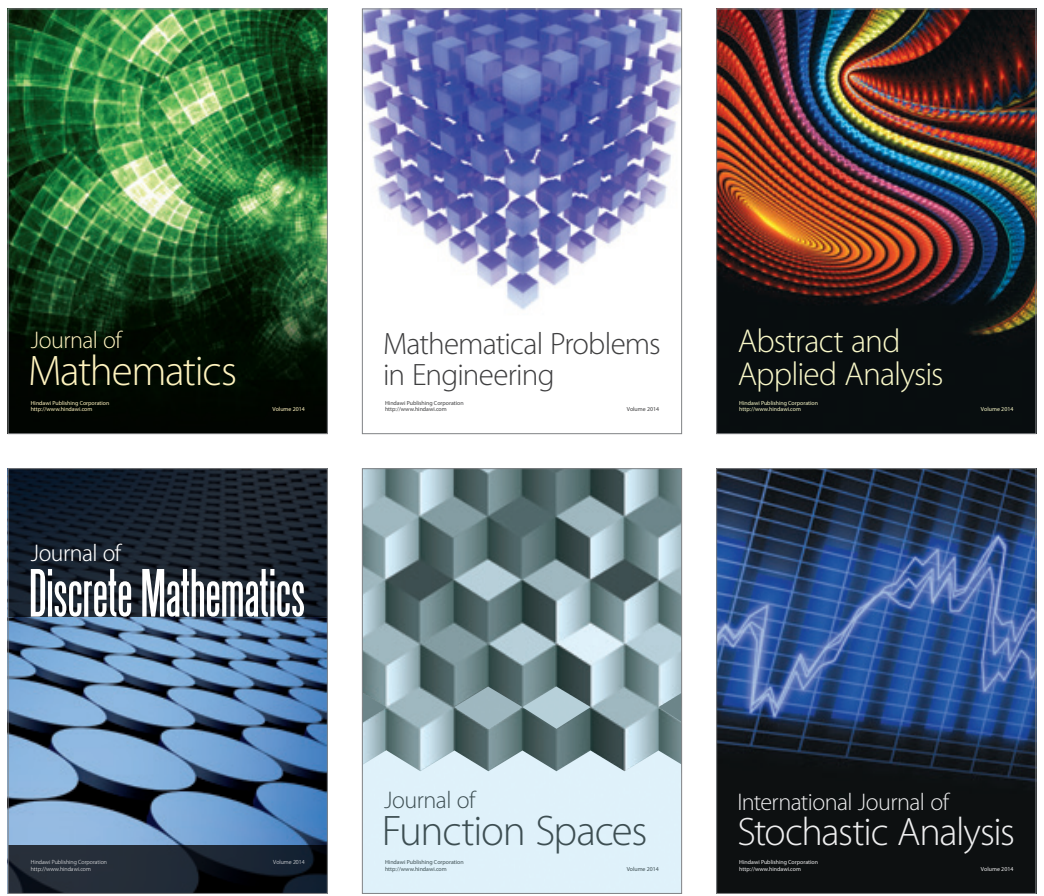

Journal of

Function Spaces

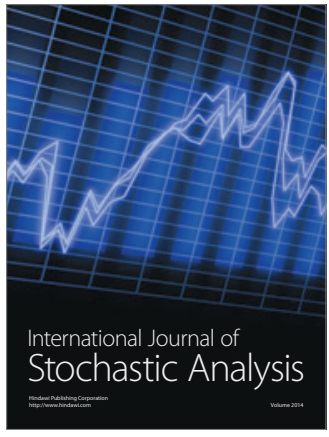

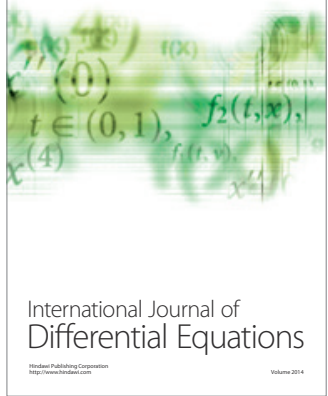
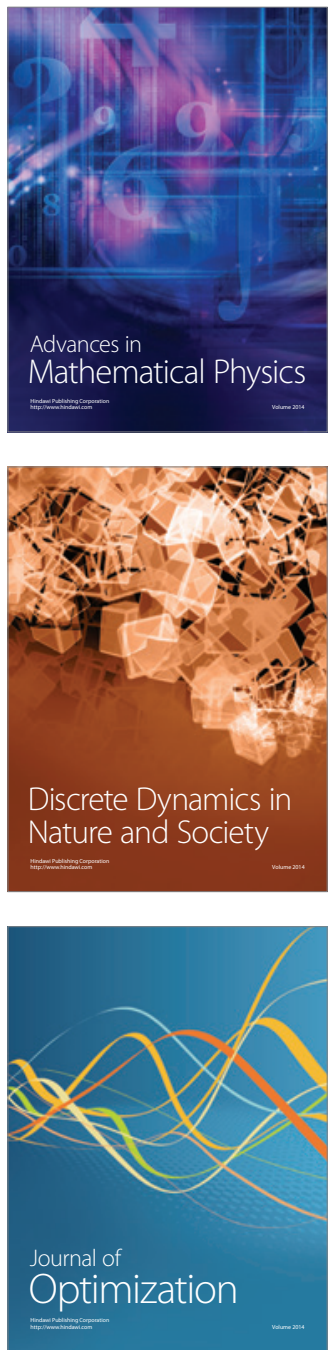